Type of the Paper (Article.)

\title{
Title Looking at quantization of a wave function, from Weber(1961), to signals from wavefunctions at the mouth of a wormhole
}

\author{
Andrew Beckwith ${ }^{1}$
}

1 Physics Department, Chongqing University, College of Physics, Chongqing University Huxi Campus, No. 44 Daxuechen Nanlu, Shapinba District, Chongqing 401331, People’s Republic of China, rwill9955b@gmai.com

\section{Abstract:}

We utilize how Weber in 1961 initiated the process of quantization of early universe fields to the problem of what may be emitted at the mouth of a wormhole. While the wormhole models are well developed, there is as of yet no consensus as to how, say GW or other signals from a wormhole mouth could be quantized, or made to be in adherence to a procedure Weber cribbed from Feynman, in 1961. In addition, we utilize an approximation for the Hubble parameter parameterized from Temperature using Sarkar's H $\sim$ Temperature relations, as given in the text . Finally after doing this we go to the Energy as $\mathrm{E}$ also $\sim$ Temperature, and from there use E (energy) as signal frequency. This gives us an idea of how to estimate frequency generated at the mouth of a wormhole.

Keywords: Minimum scale factor, cosmological constant, space-time bubble, bouncing cosmologies

\section{Introduction}

We bring up this study first a result given by Weber, in 1961 [1] as to getting an initial wavefunction given in [2], which may be able to model behavior of what happens in the mouth of a wormhole if we make the assumption given in [3] that $\mathrm{H}$ (Hubbles parameter) is proportional to Temperature, and then go to Energy $\sim$ Temperature. The last part will be enough to isolate, up to first principles a net frequency value

The behavior of frequency, versus certain conditions at the mouth of a wormhole may give us clues to be investigated later as to polarization states relevant to the wormhole [4] as well as examining what may be relevant to measurement of signals from a wormhole [5]

In doing all of this, the idea is that we are evolving from the Einstein-Rosen bridge to a more complete picture of GR which may entail a new representation of the Visser "Chronology protection" paper as in [6]

\section{Looking at the Weber book as to reformulate Quantization im-} posed in GR from

Using [1] a statement as to quantization for a would be GR term comes straight

$$
\Psi_{\text {Later }}=\int \sum_{H} e^{\left(i I_{H} / \hbar\right)\left(t, t^{0}\right)} \Psi_{\text {Earlier }}\left(t^{0}\right) d t^{0}
$$

The approximation we are making is to pick one index, so as to have'

$$
\Psi_{\text {Later }}=\int \sum_{H} e^{\left(i I_{H} / \hbar\right)\left(t, t^{0}\right)} \Psi_{\text {Earlier }}\left(t^{0}\right) d t^{0} \underset{H \rightarrow 1}{\longrightarrow} \int e^{\left(i I_{H_{F I X E D}} / \hbar\right)\left(t, t^{0}\right)} \Psi_{\text {Earlier }}\left(t^{0}\right) d t^{0}(2)
$$


This corresponds to say being primarily concerned as to GW generation, which is what we will be examining in our ideas, via using

$$
e^{\left(i I_{H_{F I X E D}} / \hbar\right)\left(t, t^{0}\right)}=\exp \left[\frac{i}{\hbar} \cdot \frac{c^{4}}{16 \pi G} \cdot \int_{\mathrm{M}} d t \cdot d^{3} r \sqrt{-g} \cdot(\mathfrak{R}-2 \Lambda)\right]
$$

We will use the following, namely, if $\Lambda$ is a constant, do the following for the Ricci scalar [8][9]

$$
\mathfrak{R}=\frac{2}{r^{2}}
$$

If so then we can write the following, namely: Eq.(3) becomes, if we have an invariant Cosmological constant, so we write $\Lambda \underset{\text { all-time }}{\longrightarrow} \Lambda_{0}$ everywhere, then

$$
e^{\left(i I_{H_{F X E D}} / \hbar\right)\left(t, t^{0}\right)}=\exp \left[\frac{i}{\hbar} \cdot \frac{c^{4} \cdot \pi \cdot t^{0}}{16 G} \cdot\left(r-r^{3} \Lambda_{0}\right)\right]
$$

Then, we have that Eq. (1) is re written to be

$$
\begin{aligned}
& \Psi_{\text {Later }}=\int \sum_{H} e^{\left(i I_{H} / \hbar\right)\left(t, t^{0}\right)} \Psi_{\text {Earlier }}\left(t^{0}\right) d t^{0} \\
& \underset{\text { at-wormhole }}{\longrightarrow} \int \exp \left[\frac{i}{\hbar} \cdot \frac{c^{4} \cdot \pi \cdot t^{0}}{16 G} \cdot\left(r-r^{3} \Lambda_{0}\right)\right] \Psi_{\text {Earlier }}\left(t^{0}\right) d t^{0}
\end{aligned}
$$

\section{Examining the behavior of the Earlier wavefunction in Eq. (6)}

[2] states a Hartle-Hawking wavefunction which we will adapt for the earlier wavefunction as stated in Eq. (6) so as to read as follows

$$
\Psi_{\text {Earlier }}\left(t^{0}\right) \approx \Psi_{H H} \propto \exp \left(\frac{-\pi}{2 G H^{2}} \cdot(1-\sinh (H t))^{3 / 2}\right)
$$

Here, making use of Sarkar [3], we set, if say $g_{*}$ is the degree of freedom allowed [9]

$$
H=1.66 \sqrt{g_{*}} T_{\text {temp }}^{2} / M_{\text {Planck }}
$$

We assume initially a relatively uniformly given temperature, that $\mathrm{H}$ is constant.

So then we will be attempting to write out an expansion as to what the Eq.(6) gives us while we use Eq. (7) and Eq. (8), with H approximately constant. If so then

\section{Methods used in Calculating Eq. (6), with interpretation of the results}

We will be considering how, to express Eq. (6) And in doing this we will be looking at having a constant value for Eq. (8). If so then

$$
\begin{aligned}
& \Psi_{\text {Later }} \\
& =\int \exp \left[\frac{i}{\hbar} \cdot \frac{c^{4} \cdot \pi \cdot t^{0}}{16 G} \cdot\left(r-r^{3} \Lambda_{0}\right)\right] \exp \left(\frac{-\pi}{2 G H^{2}} \cdot(1-\sinh (H t))^{3 / 2}\right) d t^{0}
\end{aligned}
$$

Then using numerical integration, [10],[11],[12] on page 751 of this [12] citation 


$$
\begin{aligned}
& \Psi_{\text {Later }} \underset{t_{M} \rightarrow \varepsilon^{+}}{\longrightarrow} \int_{0}^{t_{M}} e^{i \cdot(\tilde{\alpha} 1) \cdot t-(\tilde{\alpha} 2) \cdot(1-\sinh (H t))^{3 / 2}} d t \\
& \approx \frac{t_{M}}{2} \cdot\left(e^{i \cdot(\tilde{\alpha} 1) \cdot t_{M}-(\tilde{\alpha} 2) \cdot\left(1-\sinh \left(H \cdot t_{M}\right)\right)^{3 / 2}}-1\right) \\
& \tilde{\alpha} 1=\left[\frac{c^{4} \cdot \pi}{16 G \hbar} \cdot\left(r-r^{3} \Lambda_{0}\right)\right], \quad \tilde{\alpha} 2=\frac{\pi}{2 G H^{2}}
\end{aligned}
$$

Notice the terms for the $\mathrm{H}$ factor, and from here we will be making our prediction If the energy, $\mathrm{E}$, has the following breakdown

$$
\begin{aligned}
& H=1.66 \sqrt{g_{*}} T_{\text {temp }}^{2} / M_{\text {Planck }} \\
& \Rightarrow E \approx k_{B} T_{\text {Temp }} \approx \hbar \cdot \omega_{\text {signal }} \\
& \Rightarrow \omega_{\text {signal }} \approx \frac{k_{B} \cdot \sqrt{M_{\text {Planck }} H}}{\hbar \cdot \sqrt{1.66 \sqrt{g_{*}}}}
\end{aligned}
$$

The upshot is that we have, in this, a way to obtain a signal frequency by looking at the real part of Eq. (11) above, if we have a small $t$, initially (small time step)

\section{How to compare with a Kieffer solution and thereby isolate the Cosmological constant contribution}

Eq. (11) would imply an initial frequency dependence,. What we are doing next is to strategize as to understand the contribution of the cosmological constant in this sort of problem. I.e. the way to do it would be to analyze a Kieffer "dust solution" as a signal from the Wormhole. i.e. look at [13], where we assume that $t$, would be in this case the same as in Eq. (10) above. I.e. in this case we will write having

$$
\Delta \omega_{\text {signal }} \Delta t \approx 1
$$

If so then we can assume, that the time would be small enough so that

$$
\Delta t \approx \frac{\hbar \sqrt{1.66 \sqrt{g_{*}}}}{k_{B} \cdot \sqrt{M_{\text {Planck }} H}}
$$

If Eq. (13) is of a value somewhat close to $t$, in terms of general initial time, we can write

$\psi_{\tilde{n}, \lambda}(t, r) \equiv \frac{1}{\sqrt{2 \pi}} \cdot \frac{\tilde{n} !(2 \lambda)^{\tilde{n}+1 / 2}}{\sqrt{(2 \tilde{n}) !}} \cdot\left[\frac{1}{(\lambda+i \cdot t+i \cdot r)^{\tilde{n}+1}}-\frac{1}{(\lambda+i \cdot t-i \cdot r)^{\tilde{n}+1}}\right](\mathbf{1})$

Here the time $t$ would be proportional to Planck time, and $r$ would be proportional to Planck length, whereas we set

$$
\lambda \approx \sqrt{\frac{8 \pi G}{V_{\text {volume }} \hbar^{2} t^{2}}} \underset{G=\hbar=\ell_{\text {Planck }}=k_{B}=1}{\longrightarrow} \sqrt{\frac{8 \pi}{t^{2}}} \equiv \frac{\sqrt{8 \pi}}{t}
$$

Then a preliminary emergent space-time wavefunction would take the form of 


$$
\begin{aligned}
& \psi_{\tilde{n}, \lambda}(\Delta t, r) \\
& \equiv \frac{1}{\sqrt{2 \pi}} \cdot \frac{\tilde{n} ! \cdot\left(2 \cdot \sqrt{8 \pi} \cdot(\Delta t)^{-1}\right)^{\tilde{n}+1 / 2}}{\sqrt{(2 \tilde{n}) !}} \cdot\left[\begin{array}{l}
\frac{1}{\left(\sqrt{8 \pi} \cdot(\Delta t)^{-1}+i \cdot \Delta t+i \cdot r\right)^{\tilde{n}+1}} \\
-\frac{1}{\left(\sqrt{8 \pi} \cdot(\Delta t)^{-1}+i \cdot \Delta t-i \cdot r\right)^{\tilde{n}+1}}
\end{array}\right]
\end{aligned}
$$

Just at the surface of the bubble of space-time, with $t_{\text {Planck }} \propto \Delta t$, and $r \propto \ell_{\text {Planck }}$

This is from a section, page 239 of the $3^{\text {rd }}$ edition of Kieffer's book, as to a quantum theory of collapsing dust shells, . And so then we have the following procedure as to isolate out the contribution of the Cosmological constant. Namely, take the REAL part of Eq. (16) and compare it with the Real part of Eq. (10)

Another way to visualize this situation and this is a different way to interpret $\mathrm{Eq}$, (15). To do so we examine looking at page 239 of Kieffer, namely [13] where one has an expectation value to energy we can write as

$$
\langle E\rangle_{\kappa=n, \lambda}=\frac{(\kappa=n)+1 / 2}{\lambda} \underset{\lambda \approx 1 / \hbar \omega}{\longrightarrow} \hbar \omega \cdot((\kappa=n)+1 / 2)
$$

What we can do, is to ascertain the last step would be to make the Eq. (16) in a sense partly related to the simple harmonic oscillator. But we should take into consideration the normalization using that if $\hbar=\ell_{P}=G=t_{P}=k_{B}=1 \quad$ is done via Plank unit normalization[14][15]. If so, then we have that frequency is proportional to $1 / t$, where $t$ is time. I.e. hence if there is a value of $n=0$ and making use of the frequency, we then would be able to write Eq. (16) as

$$
\begin{aligned}
& \Psi_{1, \kappa=n=0} \approx \sqrt{\frac{\omega}{\pi}} \cdot\left[\frac{1}{\omega+i \cdot(t+r)}-\frac{1}{\omega+i \cdot(t-r)}\right] \\
& \text { Or, } \\
& \Psi_{2, \kappa=n=0} \approx \frac{1}{\sqrt{\pi}} \sqrt{\frac{\sqrt{8 \pi}}{t}} \cdot\left[\frac{1}{\frac{\sqrt{8 \pi}}{t}+i \cdot(t+r)}-\frac{1}{+\frac{\sqrt{8 \pi}}{t} i \cdot(t-r)}\right]
\end{aligned}
$$

With, say

$$
\omega \approx \frac{\sqrt{8 \pi}}{t}
$$

And this in a setting where we have the dimensional reset of Planck Units[14][15]

$$
\hbar=\ell_{P}=G=t_{P}=k_{B}=1
$$

\section{Formal bounding of the cosmological constant, in terms of two wavefunctions plus analysis of initial wormhole frequency values}

To do this we first of all consider the real part of Eq. (18) when $\mathrm{n}$ is set equal to zero, and the state it should be the same as the real part of Eq. (10). Then do the same with the real part of Eq. (19) and compare with Eq. (10) in order to isolate out a bound to the cosmological constant.

Doing so, leads to the following situation, and keep in mind that we are using Eq. (21) for regularization as well as setting

$$
r \equiv \widehat{B} \cdot r_{P} \underset{r_{P} \rightarrow 1}{\longrightarrow} \widehat{B}
$$


If so, then we have the following bounding as far as the value of the cosmological "constant", namely

$\Psi_{\text {Later }} \underset{t_{M} \rightarrow \varepsilon^{+}}{\longrightarrow} \int_{0}^{t_{M}} e^{i \cdot(\tilde{\alpha} 1) \cdot t-(\tilde{\alpha} 2) \cdot(1-\sinh (H t))^{3 / 2}} d t$

$\approx \frac{t_{M}}{2} \cdot\left(e^{i \cdot(\tilde{\alpha} 1) \cdot t_{M}-(\tilde{\alpha} 2) \cdot\left(1-\sinh \left(H \cdot t_{M}\right)\right)^{3 / 2}}-1\right) \approx$

$\Psi_{1, \kappa=n=0} \approx \sqrt{\frac{\omega}{\pi}} \cdot\left[\frac{1}{\omega+i \cdot(t+r)}-\frac{1}{\omega+i \cdot(t-r)}\right]$

$\tilde{\alpha} 1=\left[\frac{c^{4} \cdot \pi}{16 G \hbar} \cdot\left(r-r^{3} \Lambda_{0}\right)\right], \quad \tilde{\alpha} 2=\frac{\pi}{2 G H^{2}}$

$\tilde{\alpha} 2=\frac{\pi}{2 G H^{2}}$

We will be looking at comparing the real values of Eq. (23) in order to obtain a bound on the cosmological constant, and in doing so we have employing the following Eq.(11), Eq. (20), Eq,.(21), Eq. (22) and Eq. (23) in order to get a bound on the Cosmological constant as given by

$$
\begin{aligned}
& \Lambda_{0} \approx \tilde{B}^{-2}-\frac{16}{\pi} \cdot \tilde{B}^{-2} \cdot\left(\tilde{\alpha} 2 \cdot(1-\sinh (H \cdot \tilde{B}))^{3 / 2}\right) \\
& -\frac{16}{\pi} \cdot \tilde{B}^{-2} \cdot \cos ^{-1}\left[\frac{2 \cdot 8^{3 / 4} \cdot \pi^{1 / 4}}{8 \pi+(1+\tilde{B})^{2}}-\frac{2 \cdot 8^{3 / 4} \cdot \pi^{1 / 4}}{8 \pi+(1-\tilde{B})^{2}}\right]
\end{aligned}
$$

In doing this, taking into account the Planck units and their normalization, we also need to keep in consideration the frequency, which we will denote here as

$$
\omega_{\text {signal }} \approx \frac{k_{B} \cdot \sqrt{M_{\text {Planck }} H}}{\hbar \sqrt{1.66 \sqrt{g_{*}}}} \underset{\hbar=\ell_{P}=G=t_{P}=k_{B}=1}{\longrightarrow} \frac{\sqrt{H}}{\sqrt{1.66 \sqrt{g_{*}}}} \approx \frac{T_{\text {temperature }}}{2}
$$

Whereas what we will be doing, after we obtain a frequency of a signal near the mouth of a wormhole is to use the following scaling of frequency, near Earth Orbit from this wormhole. First if the wormhole is right at the start of the Universe[8], we use $\left(1+z_{\text {initial-era }}\right) \equiv \frac{a_{\text {today }}}{a_{\text {initial-era }}} \approx\left(\frac{\omega_{\text {Earth-orbit }}}{\omega_{\text {initial-era }}}\right)^{-1}$

$\Rightarrow\left(1+z_{\text {initial-era }}\right) \omega_{\text {Earth-orbit }} \approx 10^{25} \omega_{\text {Earth-orbit }} \approx \omega_{\text {initial-era }}$

If we are say far closer to the Earth, or the Solar system, then we would likely see[8]

$$
10 \cdot \omega_{\text {Earth-orbit-signal }} \approx \omega_{\text {wormhole-mouth-signal }}
$$

Our derivation so far is to obtain the initial signal frequency for Eq.(26) and Eq.(27). Our next task is to obtain some considerations as to the Polarization, of say GW to observe and look for, in conclusion of this document.

\section{The big picture, polarization of signals from a wormhole} mouth may affect GW astronomy investigations

We will be referencing [16] and [17]. i.e. for [16] we have a rate of production from the worm hole mouth we can quantify as 


$$
\Gamma \approx \exp \left(\omega_{\text {signal }} / T_{\text {temperature }}\right)
$$

Whereas we have from [17] a probability for "scalar" particle production from the wormhole given by

$$
\Gamma \approx \exp \left(-E / T_{\text {temperature }}\right)
$$

Whereas if we assume that there is a "negative temperature in Eq. (28) and say rewrite Eq. (29) as obeying having

$$
\left(\omega_{\text {signal }} / T_{\text {temperature }}\right) \approx\left(-E / T_{\text {temperature }}\right)
$$

This is specifying a rate of particle production from the wormhole. And so then :

Whereas what we are discussing in Eq. (28) and Eq. (29) is having a rate of , from a wormhole mouth, presumably from graviton production. If as an example, we are examining the mouth of a wormhole as being equivalent of a linkage between two black holes, or a black hole - white hole pair, we are presuming a release from the mouth of the wormhole commensurate with looking at [18] with an eye to "white holes" for a black hole model as of probability for "scalar" particle production given as, if M is the mass of the black(white) hole, $m$ is the mass of an emitted "particle", $\omega$ is frequency of emitted particles,

$$
\Gamma \propto \exp \left(-8 \pi M \cdot \omega \cdot\left[1+\frac{\beta}{4} \cdot\left(m^{2}+4 \omega^{2}\right)\right]\right)
$$

Whereas we define the parameter $\beta$ via a modified energy expression, as in [18] given by $\tilde{E}$ as a modified energy expression in [18] , [19]

$$
\tilde{E}=E \cdot\left(1-\beta \cdot\left(p^{2}+m^{2}\right)\right)
$$

Our equation (28) and (29), which are for wormholes, should encompass the same information of Eq. (31) which would be consistent with a white hole [20] [21'] the mouth of a worm hole, as would be expected from Eq. (31), whereas reviewing a linkage between black holes and white holes as may be for forming a wormhole may give more credence to the information loss criteria as given in [22]

Our next step is to ask if this permits speaking of say GW polarization in the mouth of a worm hole.

To do this, first of all, note that in [23] that the simplest version of a worm hole is one of two universes connected by a "throat" of the form of a "ball' given by $\pi b^{2}$, whereas the term $b$, is in a diagram, consigned to be the radius, or shape of the initial "ball" joining two "universes"

In the case of extending $b$ to become the "shape" of the mouth of a wormhole, we would likely be using [24] for what is called by Visser the "shape" function of the wormhole[25], whereas what we are referring to in Eq. (33) comes straight from [23]

$$
b(r)=\left[r_{0}{ }^{\gamma-1}+\gamma \cdot{\frac{(8 \pi G)^{\frac{\gamma-1}{\gamma}}}{\tilde{\tilde{\omega}}^{1 / \gamma}}}^{\gamma} \cdot\left(r^{3}-r_{0}^{3}\right)\right]^{\frac{\gamma}{\gamma-1}} \underset{r \rightarrow r_{0}}{\longrightarrow} r_{0}(33)
$$

Whereas we need to keep in mind the equation of state for pressure and density of[24]

$$
p=\tilde{\tilde{\omega}}(r) \cdot \rho
$$

The long and short of it is as follows. Following [24] we have that

$$
\rho_{\tilde{\alpha}}=\frac{M}{(4 \pi \tilde{\alpha})^{3 / 2}} \cdot \exp \left(-r^{2} / 4 \tilde{\alpha}\right)
$$


Whereas the $b$ coefficient in the case of NON commutative geometry is chosen [26] $b(r)=\frac{2 r_{s}}{\sqrt{\pi}} \cdot \hat{\gamma}\left(\frac{3}{2}, \frac{r^{2}}{4 \tilde{\alpha}}\right)$

$\equiv \frac{2 r_{s}}{\sqrt{\pi}} \cdot\left(\frac{r^{2}}{4 \tilde{\alpha}}\right)^{3 / 2} \cdot \tilde{\Gamma}(3 / 2) \cdot e^{-3 / 2} \cdot \sum_{k=0}^{\infty}\left(\frac{\left(\frac{r^{2}}{4 \tilde{\alpha}}\right)^{k}}{\tilde{\Gamma}((3 / 2)+k+1)}\right)$ tion [27].

This is called the incomplete lower gamma function, with $\tilde{\Gamma}$ being a gamma func-

From here, using that Eq. (36) is to be included in the following metric, as given by

The coefficient $[\tilde{\alpha}]=\left[r^{2}\right]$ in terms of dimensional analysis is chosen so that the dimensions of $[\tilde{\alpha}]=\left[r^{2}\right]$ are chosen to contain $\mathrm{M}$ as mass in a wormhole. i.e. the denominator of Eq. (35) $(4 \pi \tilde{\alpha})^{3 / 2}$ is chosen so that $M$ is within the volume of space so subscribed,. And this is for line element[26]. With Eq. (35) fully described in [28] and [26]

$$
d S^{2}=-\exp (-2 \Phi(r)) d t^{2}+\frac{d r^{2}}{1-b(r) / r}+r^{2} \cdot\left(d \theta^{2}+\left(\sin ^{2} \theta\right) d \varphi^{2}\right)(37)
$$

If we refer to black holes, with extra dimension, $n$, of Planck sized mass, we have a lifetime of the value of about

$$
\begin{aligned}
& \tau \sim \frac{1}{M_{*}}\left(\frac{M_{\mathrm{BH}}}{M_{*}}\right)^{\frac{n+3}{n+1}} \underset{M_{\mathrm{BH}} \approx M_{\text {Planck }}}{\longrightarrow} 10^{-26} \mathrm{sec} \text { onds } \\
& M_{*} \approx \text { is the low energy scale, }
\end{aligned}
$$

which could be as low as a few TeV,

The idea would be that there would be $\mathrm{n}$ additional dimensions, as given in Eq,.(38) which would then lay the door open to investigating [29] and [30] in terms of applications, with [30] of additional polarization states to be investigated, as to signals from the mouth of the wormhole. We will next then go into some predictions into first, the strength of the signals, the frequency range, and several characteristics as to the production rate of Planck sized black holes which conceivably could get evicted by use of Eq. (28), in terms of what could be observed via instrumentation.

\section{A First order guess as to the rate of production of Planck sized} black holes through a wormhole, using Eq.(28)

In order to do this, we will be estimating that the temperature would be of the order of Planck temperature, i.e. using ideas from [30] and [31]

$$
\frac{\omega_{p}}{T_{p}} \equiv \frac{\sqrt{G k_{B}^{2}}}{\hbar} \underset{\hbar=G=k_{B}=1}{\longrightarrow} 1
$$

If so, then there would be to first order the following rate of production

$$
\Gamma_{\text {rate-of-production }} \approx e \approx 2-3
$$

Some of the considerations given in this could be related to [32] as an afterthought whereas the author in [33] estimated for an LHC that there would be about 3,000 gravitons produced per second. Assuming a figure from [34] as to the percentage of black hole mass decaying into gravitons, i.e. [34], i.e,. 1/1000 of the mass of a Planck sized black hole would delve into gravitons, so if one had 3000 gravitons produced per second, as measured on Earth, one would likely have $2-3$ black holes, of mass of about $10^{\wedge}-5$ 
grams per black hole, producing say $10^{\wedge} 57$ gravitons, produced per black hole of mass about $10^{\wedge}-62$ grams per black hole [35]

Having said, that what about frequencies ? Here, if we have a wormhole throat of about 2-3 Planck lengths in diameter, with a frequency of emitted gravitons of about $10^{19} \mathrm{GHz}$ initially, it is realistic, using the following, to expect in many cases a redshift downscaling of frequencies of about $10^{\wedge}-18$, if the worm holes are close to the initial near singularity, so then that we could be looking at approximately 10 to $12 \mathrm{GHz}$, on Earth, for frequencies, of initially about $10^{\wedge} 19 \mathrm{GHZ}$. So then note at inflation we have

$$
\begin{aligned}
& \left(1+z_{\text {initial-era }}\right) \equiv \frac{a_{\text {today }}}{a_{\text {initial-era }}} \approx\left(\frac{\omega_{\text {Earth-orbit }}}{\omega_{\text {initial-era }}}\right)^{-1} \\
& \Rightarrow\left(1+z_{\text {initial-era }}\right) \omega_{\text {Earth-orbit }} \approx 10^{25} \omega_{\text {Earth-orbit }} \approx \omega_{\text {initial-era }}
\end{aligned}
$$

In our situation, the figure would likely be instead of $10^{\wedge} 25$ times Earth orbit detected frequency, something closer to $10^{\wedge} 18$ to $10^{\wedge} 19$ times Earth orbit GW frequencies detected as given by [36] /The relative GW strength of the signal, if one uses [36] while assuming approximately 10 to $12 \mathrm{GHz}$, for initially about $10^{\wedge} 19 \mathrm{GHz} \mathrm{GW}$ signals would be about $h \sim ~ ' 10^{\wedge}-26$ and this could change an order of magnitude given instrument sensitivity. In any case it would be well worth our while to look closely at [37][38][39][40] for additional clues and insights to consider while commencing this investigation. As well as details given in [41]

References

1. J. Weber, "General Relativity and Gravitational Waves", Dover Publications, Incorporated, Mineola, New York, USA, 2004

2. H.Q. Lu, W. Fang, Z.G. Huang, P.Y. Ji," The consistent result of cosmological constant from quantum cosmology and inflation with Born-Infeld scalar field", Eur. Phys. J. C 55, 329-335 (2008)

3. Sarkar, Utpal, "Particle and Astroparticle Physics”, Taylor \& Francis Group, New York City, New York, USA, 2008

4. Arkadii A. Popov, Sergey V. Sushkov," Vacuum polarization of a scalar field in wormhole spacetimes”, https://arxiv.org/abs/gr-qc/0009028, Phys.Rev. D63 (2001) 044017

5. DeBenedictis, Andrew \& Das, A. (2001). "On a General Class of Wormhole Geometries". Classical and Quantum Gravity. 18 (7):1187-1204. arXiv:gr-qc/0009072. Bibcode:2001CQGra..18.1187D. CiteSeerX 10.1.1.339.8662. doi:1 $\underline{0.1088 / 0264-9381 / 18 / 7 / 304}$. S2CID 119107035

6. Einstein, Albert \& Rosen, Nathan (1935). "The Particle Problem in the General Theory of Relativity". Physical Review. 48 (1): 73. Bibcode:1935PhRv...48...73E. doi:10.1103/PhysRev.48.73

7. Matt Visser, “The quantum physics of Chronology protection”, https://arxiv.org/abs/gr-qc/0204022

8. A. Lightman, W. Press, R. Price, S. Teukolsky,"Problem book in relativity and Gravitation", Princeton University Press, Princeton, New Jersey, USA, 1975

9. E. Kolb, M. Turner, "The Early Universe”, in Frontiers in Physics Lecture Note series, Addison-Wesley Publishing Company, Menlo Park, California, USA, 1990

10.McCracken D.D. and Dorn W.S., Numerical Methods and FORTRAN Programming with application in engineering and science. - Moscow: Publishing House Mir, 1977. - 584 p. 
11.Davis, Philip J., , Philip Rabinowitz ,"Methods of Numerical Integration: Second Edition “, Dover publishers, New York City, NY, USA, 2007

12. Daniel Zwillinger, CRC Standard Mathematical Tables and Formulae, $31^{\text {st }}$ edition", Chapman and Hall/CRC, Boca Raton, LA, USA, 2003

13. Klaus Kieffer, "Quantum Gravity, 3 $3^{\text {rd }}$ edition”, Oxford Science Publications, Oxford University Press, Oxford, United Kingdom, 2012

14. Barrow, John, D. "The Constants of Nature, from Alpha to Omega- The numbers that encode the deepest secrets of the Universe", New York City, New York, Pantheon Books, 2002

15. Planck, Max (1899). "Über irreversible Strahlungsvorgänge". Sitzungsberichte der Königlich Preußischen Akademie der Wissenschaften zu Berlin (in German). 5: 440-480. pp. 478-80 contain the first appearance of the Planck base units other than the Planck charge, and of Planck's constant, which Planck denoted by $b$. $a$ and $f$ in this paper correspond to $\underline{k}$ and $\underline{G}$ in modern usage.

16. Prado Martın-Moruno and Pedro F Gonzalez-Diaz, "Thermal radiation from Lorentzian traversable wormholes", Spanish Relativity Meeting (ERE 2010): Gravity as a Crossroad in Physics, Journal of Physics: Conference Series 314 (2011) 012037 ; https://iopscience.iop.org/article/10.1088/1742-6596/314/

17. Ganim Gecim and Yusuf Sucu, "Quantum Gravity Correction to Hawking Radiation of the -Dimensional Wormhole", Advances in High Energy Physics, vol. 2020, Article

ID 7516789, 10 pages, 2020. https://www.hindawi.com/journals/ahep/2020/7516789/

18. Deyou Chen , Houwen Wu , Haitang Yang and Shuzheng Yang," Effects of quantum gravity on black holes", https://arxiv.org/pdf/1410.5071.pdf, Int. J. Mod. Phys. A 29, 1430054 (2014)

19. W. Greiner, Relativistic Quantum Mechanics: Wave Equation, Springer-Verlag, 2000

20. A. Retter \& S. Heller (2012). "The revival of white holes as Small Bangs". New Astronomy. 17 (2): 73-75. arXiv:1105.2776. Bibcode:2012NewA...17...73R. doi:10.1016/j.newast.2011.07.003. S2CID 118505127

21. Nikodem J. Popławski (2010). "Radial motion into an Einstein-Rosen bridge". Physics Letters B. 687 (2-3): 110-113. arXiv:0902.1994. Bibcode:2010PhLB..687..110P. doi:10.1016/i.physletb.2010.03.029. S2CID $\underline{5947253 .}$

22. Samir D. Mathur, "Solving the information loss Paradox" https://indico.cern.ch/event/37753/contributions/1802123/attachments/752926/1032904/mathur1.pdf

23. J.A. Gonzalezx, F.S. Guzman, and O Sarbach, “On the Instability of Spherically symmetric wormholes supported by a Ghost Scalar Field", pp 208-216, of "Gravitation and Cosmology-Proceedings of the Third International Meeting, Morlia, Mochoacan, Mexico, May 26-30, 2008, AIP Conference proceedings, 1083, American Institute of Physics, Melville, NY, USA, 2009

24. R. Garranattini and F.S.N. Lobo, "Self-substained Transversable Wormholes", pp 111-135 , of Fundamental Theories of Physics, Volume 189, "Wormholes, Warp Drives and Energy Conditions", with Francisco S.N. Lobo as editor, Springer Nature publishing company, Cham, Switzerland, 2017

25. Matt Visser, "Lorentzian Wormholes" from Einstein to Hawking", AIP press, as done by Springer Verlag Press, 1996, New York City, New York, USA

26. Milton Abramowitz and Irene Stegun, "Handbook of Mathematical Functions: with Formulas, Graphs, and Mathematical Tables "(Dover Books on Mathematics) Paperback - June 1, 1965, New York City, New York, USA

27. Wittaker, E.T, Watson, G.N. "A course of Modern Analysis" Cambridge University Press, Cambridge, UK, 1927 
28. Nicolini, P., Smailagic, A.,Spallucci, E. " Non commutative geometry inspired Schwarzschild Black Hole”, Physics Letters B., 2006;632:547

29. Kingman Cheung, "Black hole production and extra large dimensions", https://arxiv.org/abs/hep-ph/0110163 Phys.Rev.Lett.88:221602,2002

30. Fang-Yu Li, Hao Wen, Zhen-Yun Fang, Di Li, Ton-Jie Zhang," Electromagnetic counterparts of high -frequency Gravitational waves having additional polarization states; distinguishing and probing tensor-mode, vector-mode, and scalar-mode gravitons" Eur. Phys. J. C (2020) 80:879, https://arxiv.org/abs/1712.00766

31. B.J. Carr, "Primordial Black Holes-Recent developments" https://arxiv.org/abs/astro-ph/0504034

32. Sokolov, A.V., Pshirkov, M.S. Possibility of hypothetical stable micro black hole production at future 100 TeV collider. Eur. Phys. J. C 77, 908 (2017).

https://doi.org/10.1140/epjc/s10052-017-5464-7

33. Andrew Beckwith," Bounds upon Graviton Mass - using the difference between graviton propagation speed and HFGW transit speed to observe post-Newtonian corrections to gravitational potential fields", https:/citeseerx.ist.psu.edu/viewdoc/download?doi=10.1.1.302.8755\&rep=rep1\&type=pdf

34. Bernard Carr, Elizabeth Winstanley, and Xavier Calmet, "Quantum Black Holes”, Springer Briefs in Physics, Springer- Nature, Cam, Switzerland, 2013

35. Clifford M. Will," Bounding the mass of the graviton using gravitational-wave observations of inspiralling compact binaries", Physical Review D. 57 (4): 2061-2068. arXiv: $\underline{\text { gr-qc/9709011. }}$

36. michele.maggiore, "Gravitational Waves, Volume 1, Theory and Experiment" Oxford University Press, Oxford, UK, 2008

37. Hawking, Stephen \& Ellis, G. F. R. (1973). The Large Scale Structure of Space-Time. Cambridge: Cambridge University Press

38. Shin'ichi Nojiri, S.D. Odintsov, V.K. Oikonomou, Tanmoy Paul, "Nonsingular bounce cosmology from Lagrange multiplier F(R) gravity", Phys. Rev. D 100, 084056 - Published 25 October 2019; https://arxiv.org/abs/1910.03546

39. Wang, Qingdi; Zhu, Zhen; Unruh, William G. (2017). "How the huge energy of quantum vacuum gravitates to drive the slow accelerating expansion of the Universe". Physical Review D. 95(10): 103504. arXiv: 1703.00543

40. Astier, Pierre (Supernova Legacy Survey); Guy; Regnault; Pain; Aubourg; Balam; Basa; Carlberg; Fabbro; Fouchez; Hook; Howell; Lafoux; Neill; Palanque-Delabrouille; Perrett; Pritchet; Rich; Sullivan; Taillet; Aldering; Antilogus; Arsenijevic; Balland; Baumont; Bronder; Courtois; Ellis; Filiol; et al. (2006). "The Supernova legacy survey: Measurement of $\Omega_{\mathrm{M}}, \Omega_{\wedge}$ and W from the first year data set". Astronomy and Astrophysics. 447 (1): 31-48. arXiv:astro-ph/0510447

41. Pisen Chen, "Resonant Photon-Graviton Conversion, 'From Earth To heaven', SLAC PUB 6666 September 1994 (T/E/A); https://www.slac.stanford.edu/pubs/slacpubs/6500/slac-pub-6666.pdf 